\title{
ILCEA
}

Revue de l'Institut des langues et cultures

d'Europe, Amérique, Afrique, Asie et Australie

$15 \mid 2012$

Les mots de la crise

\section{La langue russe à l'épreuve de la crise économique de 2007-2009 : les apports linguistiques au travers de la presse}

Testing times for the Russian language: the 2007-2009 economic crisis and its linguistic contributions as seen through the press

\section{Valéry Kossov}

\section{OpenEdition}

\section{Journals}

Édition électronique

URL : https://journals.openedition.org/ilcea/1195

DOI : 10.4000/ilcea.1195

ISSN : 2101-0609

Éditeur

UGA Éditions/Université Grenoble Alpes

Édition imprimée

ISBN : 978-2-84310-220-2

ISSN : 1639-6073

\section{Référence électronique}

Valéry Kossov, «La lanque russe à l'épreuve de la crise économique de 2007-2009 : les apports

linguistiques au travers de la presse ", ILCEA [En ligne], 15 | 2012, mis en ligne le 30 janvier 2012, consulté le 21 septembre 2021. URL : http://journals.openedition.org/ilcea/1195 ; DOI : https:// doi.org/10.4000/ilcea.1195

Ce document a été généré automatiquement le 21 septembre 2021.

(C) ILCEA 


\section{La langue russe à l'épreuve de la crise économique de 2007-2009: les apports linguistiques au travers de la presse}

Testing times for the Russian language: the 2007-2009 economic crisis and its linguistic contributions as seen through the press

Valéry Kossov

\section{NOTE DE L'AUTEUR}

L'auteur exprime ici sa gratitude envers les deux relecteurs anonymes de ce travail et tient à les remercier pour leurs observations et suggestions très constructives.

Durant ces deux dernières décennies, l'économie russe, du fait de la transition d'une économie planifiée et administrée à une économie de marché avec ses libertés, mais aussi ses limites et contraintes, a été confrontée à un grand nombre de difficultés incontournables. Sur ce fond général de difficultés économiques, au moins trois périodes ont été présentées dans l'espace médiatique russe comme des crises économiques: d'abord l'année 1992, point de départ des réformes libérales, puis 1998, année du krach financier russe, et enfin la crise financière actuelle qui recouvre la période allant d'août 2007 à décembre 2009. Nous avons déjà eu l'occasion ${ }^{1}$, d'analyser les interactions et les rapports d'interdépendance des discours politique et médiatique sur la crise en Russie. Or, il s'avère que le discours sur la crise a eu un impact important par ses apports au lexique économique ou même au langage courant. C'est pourquoi, les questions que nous analysons ici concernent les processus d'appropriation des néologismes apportés par les crises. 
Après un détour rapide vers les néologismes des années 1992 et 1998, nous nous arrêterons plus longuement sur les représentations dans la presse russe de la dernière crise financière mondiale de 2007-2009, afin d'étudier les néologismes, pour la plupart des anglicismes qui ont été introduits dans la langue russe. Comment ces néologismes sont-ils formés et intégrés par la langue ? Quelle est la fréquence de leur utilisation dans la presse en fonction des événements relatifs à la crise ? Y a-t-il un lien de dépendance entre la fréquence d'utilisation des néologismes et leur appropriation par la langue?

Actuellement le paysage journalistique en Russie est caractérisé à la fois par une grande variété d'éditions orientées vers des groupes sociaux et des publics de lecteurs divers et aussi par leur degré de loyauté par rapport au pouvoir politique et à cet égard, nous pouvons établir trois catégories générales. Parmi les éditions dites "généralistes", se distinguent des journaux, comme Rossiyskaya Gazeta dépendant directement du Kremlin et reflétant le point de vue strictement officiel, tout en se permettant quelques critiques occasionnelles des pouvoirs régionaux ou locaux. D'autre part, il existe des journaux qui sont ouvertement opposés au régime politique actuel et proposent à leurs lecteurs un discours critique sur l'action du gouvernement. Enfin, un nombre important de journaux se trouvent sous la pression du pouvoir mais rapportent aux lecteurs les erreurs et incohérences de la politique de l'État et proposent leur analyse des événements sociopolitiques et économiques sortant de l'attitude de «bien-pensance» et du conformisme.

Notre corpus pour cette étude sera donc constitué de titres de ce genre de presse généraliste ${ }^{2}$, c'est-à-dire adressée à un public large, mais contenant des articles destinés à un lectorat non-spécialiste intéressé par une analyse compétente des questions économiques et financières. Ce choix de la presse se justifie, car en raison de son caractère universel et de son public-cible vaste, elle reflète d'une manière plus globale l'évolution des "mots de la crise ${ }^{3}$ ». On a choisi trois éditions en "opposition tacite » au pouvoir, le quotidien Kommersant et ses deux suppléments hebdomadaires, les magazines Vlast' et Den'gui, l'hebdomadaire Profil, et les quotidiens Izvestia et Novye Izvestia, qui ont été considérés comme sources prioritaires. En effet, sans être en conflit ouvert avec le pouvoir (comme le journal Novaya gazeta), ils offrent à leurs lecteurs des points de vue souvent divergents de la ligne politique générale, avec le souci d'aborder les problèmes sous un angle critique et sans parti pris. Cette politique éditoriale se reflète dans le style général des articles, d'où se dégage un discours qu'on pourrait nommer "discours d'expert », destiné toutefois à un public intéressé par, mais non-spécialiste du domaine de la finance. C'est cependant ce public qui peut nous servir d'indicateur pour mesurer la profondeur de l'ancrage des néologismes dans la langue car il constitue la cible potentielle pour le discours expert de la presse.

\section{Les crises et les néologismes}

Sans vouloir s'arrêter longtemps sur les définitions du terme " néologisme ", nous nous appuierons, dans cet article, sur la notion du néologisme en tant qu'une unité lexicale nouvelle dans la langue et correspondant à un sentiment spécifique de "nouveauté " chez les locuteurs. C'est davantage la néologie morphosémantique constituée par les emprunts et étudiée en synchronie qui nous intéressera dans ce travail. Un lien entre l'apparition des néologismes et les changements sociaux mis en relief dans les définitions 
soviétiques sera également un critère d'évaluation des «mots de la crise » dans notre démarche méthodologique.

Comme tant d'autres événements sociétaux (révolutions, coups d'État, changements de systèmes politiques, etc.) les crises économiques en Russie ont toujours été une source d'enrichissement de la langue, soit au niveau de la terminologie spécialisée qu'on retrouve dans le discours d'expert, soit au niveau sémantique, lorsque des mots du langage courant changent de sens dans le contexte de la crise, soit dans la syntaxe et la phraséologie. Certes, en grande majorité les néologismes issus des crises économiques sont d'origine anglaise, les crises elles-mêmes étant associées le plus souvent dans la presse russe au modèle du libéralisme anglo-saxon. Toutefois, le processus d'appropriation des anglicismes par la langue russe est soumis à un certains nombre de critères, et la simple utilisation d'un terme d'origine anglaise dans le discours d'expert ne signifie pas toujours son passage définitif dans la langue russe.

Le caractère synthétique du système linguistique du russe implique que l'utilisation d'un néologisme doit se faire conformément aux règles de la formation des mots, avec l'ajout de suffixes, préfixes, flexions. L'appropriation d'un néologisme suppose également son adaptabilité aux différentes catégories grammaticales pour permettre aux néologismes de remplir des fonctions différentes dans la phrase. Enfin, le néologisme entre définitivement dans la langue lorsqu'il est adopté par les usagers, qui l'emploient couramment dans des expressions figées ou imagées. Il commence alors à remplir des fonctions extralinguistiques et pragmatiques, en reflétant l'humour, l'ironie ou d'autres sentiments. Parmi ce type de néologismes russifiés, il faut accorder une attention particulière à certains emprunts que l'on peut considérer comme des mots-repères, qui ont marqué une époque et sont employés toujours en association avec le moment de leur apparition.

Ce sont les deux premières crises économiques en Russie, en 1992 et en 1998, qui ont produit, outre un certain nombre de termes économiques empruntés, ces mots-repères qui désormais font référence, aussi bien qu'à l'époque de leur apparition, à un certain nombre de figurants, hommes politiques ou économistes qui pendant cette période, étaient au devant de la scène politique russe.

Ainsi, les réformes de 1992 avec comme acteurs politiques principaux Egor Gaïdar et Anatoly Tchoubaïs, sont caractérisés par les mots : ШоКОВАЯ ТЕРАПИЯ (thérapie de choc) et ВАУЧЕP (voucher) se rapportant respectivement à la libéralisation des prix (Gaïdar) et les privatisations (Tchoubaïs). Au moment de leur apparition, dans le discours politique d'abord, et dans la presse ensuite, aucune des deux locutions, la première étant un calque de l'anglais, la seconde une transcription, n'avait un sens bien clair pour la population russe. Le processus de leur russification s'est accompagné d'événements qui leur donnaient des significations nouvelles ayant un rapport de plus en plus étroit avec les réalités russes de cette époque. La formation, sur la base de ces emprunts, d'expressions aux connotations ironiques, s'explique d'une part par leur sens encore flou au début de leur utilisation et, d'autre part, par leur adoption dans la langue en tant que mots faisant référence à la crise de 1992 : « ШоК БЕЗ ТЕРАПИИ ( (un choc sans thérapie ${ }^{4}$ ), «ВЛОЖИТЬ/ ЗАСУНУТЬ ВАУЧЕР » (placer/introduire son voucher). L'utilisation de ces néologismes s'avère fort prisée par la suite, elle permet toutes sortes d'allusions expressives comme, par exemple, « ЗАСУНЬТЕ СВОЙ ВАУЧЕР КУДА ПОДАЛЬШЕ!» (Mettez votre voucher là où je pense !). 
La crise de 1998 a également contribué à l'enrichissement de la langue en produisant de nouveaux mots-repères, parmi lesquels on distingue avant tout « ДЕФОЛТ » (transcription de l'anglicisme default). Ce mot entre également dans la langue à travers le discours politique où il perd son sens d'origine (cessation de paiements, défaillance) tout en restant une « coquille vide " pour la majorité de la population russe. En fin de compte, ce mot se remplit d'un sens plus large qui, sans se limiter à la défaillance financière de l'État russe, englobe l'ensemble des conséquences pour la population du krach financier, mais aussi des problèmes de la vie quotidienne. Cet élargissement sémantique permet de produire des expressions comme « полный дЕФолт » utilisé comme un euphémisme en lieu et place d'une expression grossière relevant du mat, registre lexical spécifique des invectives taboues.

Peut-on trouver dans la langue d'aujourd'hui des exemples du même genre se rapportant à la dernière crise économique? Avant de nous pencher sur la question des apports linguistiques de la dernière crise, nous nous proposons de dresser un cadre chronologique qui nous permettra de suivre l'évolution des représentations de la crise et les fluctuations d'emploi des mots spécifiques de la crise dans la presse russe entre 2007 et 2009 .

\section{Représentations de la crise dans la presse : aperçu chronologique}

Le début de la crise financière aux États-Unis, en février 2007, se fait sentir à la bourse de Moscou par la baisse de l'indice russe RTS et, plus tard, en juillet 2007, les indices boursiers russes se montrent très instables. Cependant, dans la presse, on n'évoque que de façon indirecte l'impact de la crise financière américaine sur le secteur des finances en Russie.

D'une manière générale, la crise apparaît comme peu probable en Russie tout au long de l'année 2007. Les problèmes de l'instabilité des indices de la bourse de Moscou et le départ des capitaux sont évoqués dans la presse sans qu'on en tire des conclusions à long terme pour l'économie russe. En revanche, dans la représentation de la crise financière américaine, la presse économique s'en donne à cœur joie sans lésiner sur les métaphores et les références au passé de la Russie. Notamment, on évoque «le mardi noir des marchés de valeurs » en faisant allusion au mardi noir de 1995, où le rouble a perdu un tiers de sa valeur en Russie en une journée (о. КочЕВА, П. чуВИЛЯЕВ, 2007). Les métaphores développées se rapportent souvent à la thématique de "l'ours » et du « taureau », ces symboles américains au départ, du jeu boursier à la baisse et à la hausse, qui se sont banalisés dans le monde de la finance internationale. Comme on le voit dans l'exemple de l'article intitulé «Les rêves de l'ours » (чувиляЕВ, 2007), les métaphores animalières permettent de transposer le langage souvent codé des financiers afin de présenter les informations d'une manière plus vivante et animée.

La fin de l'année 2007 et le début de 2008 se déroulent dans une ambiance relativement stable, aussi bien sur les marchés financiers que dans la presse. Le calme des marchés s'explique par certains facteurs internes liés aux résultats assez prévisibles des élections législatives de décembre 2007 (la déclaration d'intention de Vladimir Poutine de prendre la tête du parti du pouvoir « Russie Unie » et d'accepter le poste de Premier ministre en cas d'élection de son successeur, déjà annoncé, Dmitri Medvedev au poste de président). 
Cependant, les facteurs extérieurs, notamment les conséquences de la crise des subprimes pour tous les secteurs de la finance, la publication des indices macroéconomiques américains, les informations sur les pertes des grands groupes industriels, provoquent l'effondrement des marchés de valeurs mondiaux. La Russie n'y fait pas exception. La presse, malgré une certaine dose d'humour censée dédramatiser (АСКЕР-ЗАДЕ, 20085), se montre de plus en plus inquiète de la situation sur les marchés financiers en Russie à partir de janvier 2008.

L'année 2008 commence par la confirmation de la tendance à la baisse des indices russes à partir du mois de mai (ИлЛАРИОНОВ, 2008: 4). La fragilité du marché des valeurs en Russie ainsi que la dépendance de l'économie russe des décisions politiques deviennent évidentes en juillet 2008 lorsque, après une attaque verbale du nouveau Premier ministre Vladimir Poutine contre la société Mechel, la cote des actions des entreprises russes connaît une baisse considérable.

Comme les problèmes des indices boursiers ne concernent pas globalement les larges couches populaires d'électeurs, les autorités russes, préoccupées avant tout par les élections présidentielles de mars 2008, ne manifestent pas d'inquiétude particulière. Qui plus est, le nouveau président, Medvedev, dans un discours certes beaucoup plus modéré que celui de son prédécesseur, met une fois de plus en cause l'efficacité du système financier américain et son influence sur les systèmes économiques des autres pays. Lors du forum économique de Saint-Pétersbourg en juin 2008, il propose de transférer le centre financier mondial en Russie, arguant de la position dominante de la Russie sur le marché de l'énergie, de la hausse des prix agricoles, et présentant son pays comme économiquement capable d'assumer ce statut, tandis que les États-Unis auraient surestimé leurs capacités (БУТУРлин, ШАПОВАЛОВ, 2008). Certes, le titre et les expressions relatant cette intervention dans Kommersant contiennent une part d'ironie par rapport aux propos du Président : « Dmitri Medvedev a creusé jusqu'aux racines de la crise financière. Le monde a pâti du manque d'influence de la Russie sur l'économie globale. » Il est tout à fait probable que cette intervention de Medvedev n'était qu'à moitié sérieuse et que la provocation était calculée. Cela ressemble fort à une nouvelle ${ }^{6}$ tactique discursive du pouvoir en temps de crise, qui consiste à se montrer très offensif. $\mathrm{Au}$ lieu de rassurer la population sur la situation économique, Medvedev avance ses pions, en posant, dès le départ, le principe de la "puissance économique » de la Russie comme un fait accompli, qui devrait donc lui assurer son "rôle dirigeant» dans le domaine financier.

Dès son entrée en fonction Medvedev tente de rassurer le monde des affaires. Imitant son prédécesseur, il introduit dans son discours des expressions simples et imagées, qui sont reprises par la suite dans la presse et appliquées, à titre d'illustration, à des situations différentes. Cependant, l'imitation ne parvient que rarement à dépasser l'original, et en 2008 Medvedev est encore en train d'investir sa fonction et de forger son image. Un élément parmi d'autres dans le discours du tandem Poutine/Medvedev permet de le distinguer de celui de ses prédécesseurs, c'est le choix des néologismes qui se veulent «populaires» ou "patriotiques », c'est-à-dire d'origine russe, non-étrangère. Contrairement aux dirigeants russes des années 1990, le tandem au pouvoir actuellement évite soigneusement l'abus des anglicismes car ce sont justement eux qui ont joué un mauvais tour aux réformateurs des années 1990, en rendant leur discours trop atone, complexe, peu émotionnel et surtout peu patriotique pour de larges couches populaires. Les néologismes d'origine russe de Poutine-Medvedev sont, en revanche, un facteur de 
sympathie et de popularité car, adressés au grand public, ils sont à la fois compréhensibles et inventifs : « КОШМАРИТЬ БИЗНЕС, РЕШАЛЫ, ПОСАДКИ », etc.

Certes, les pratiques discursives politiques n'ont pas le pouvoir de dissimuler l'évolution de la crise et le moment venu il faut trouver les moyens d'expliquer et de reconnaître la réalité. La véritable reconnaissance de la crise arrive en août 2008, après la guerre de cinq jours contre la Géorgie en Ossétie du Sud. En effet, aussi bien la Banque mondiale que les autorités russes évoquent le début d'une crise provoquée par des emprunts excessifs du secteur privé, dans une situation où se dégradent les conditions du commerce extérieur et des emprunts à l'étranger, et où s'intensifie le départ des capitaux ${ }^{7}$. Pendant le conflit, les autorités n'évoquent qu'épisodiquement les difficultés financières, laissant cette question à la presse qui, elle, souligne l'aggravation des crises par les guerres en général, et plus particulièrement dans les conditions actuelles de l'économie globale (МИНАЕВ, 2008).

En octobre 2008, la crise financière a touché l'ensemble du système bancaire russe, ce qui contraint le gouvernement, d'une part à trouver les moyens d'accorder des crédits aux banques en difficulté, et d'autre part à procéder à des réductions du budget fédéral pour l'année 2009.

En novembre 2008, la crise s'étend au secteur industriel et devient une crise économique. La situation est aggravée pour la Russie par la baisse du prix du pétrole qui descend au dessous de 50 dollars le baril et provoque par conséquent la baisse du PIB. Ceci amène Vladimir Poutine à créer une commission gouvernementale anticrise sous la responsabilité d'un des vices premiers ministres, Igor' Chouvalov.

En même temps le discours du pouvoir relayé par la presse cultive une image protectrice de l'État, qui a préparé à ses citoyens un «coussin» ou «airbag» (ПодушкА БЕЗОПАСНОСТИ) financier sous forme de toutes sortes de réserves (КАЛЕДИНА, ЧАЙКА, 2008). Les comparaisons avec le passé permettent de court-circuiter les rumeurs sur la crise et d'éviter la panique dans la population. Cela laisse également aux autorités la liberté de tester certaines idées dans le discours avant de prendre des mesures, et ensuite de prendre des mesures sans les afficher, afin de n'en évoquer les résultats que s'ils sont positifs.

C'est ainsi que l'image de la Russie comme un «îlot de stabilité » a été dessinée par le ministre des finances Alexey Koudrine lors du Forum économique de Davos en janvier 2008 (ЧАЙКА, 2008). Cette idée semble assez attrayante et culturellement proche pour une certaine partie des Russes, depuis la théorie $\mathrm{du} \mathrm{XVI}^{\mathrm{e}}$ siècle associant Moscou à la troisième Rome. Elle est vite relayée par la presse proche du pouvoir, qui propose dans ses titres tout un choix d'expressions quasi-anachroniques, nous renvoyant aux titres de la presse soviétique qui reflétaient la position du pays - grande puissance : «La Russie tend une main secourable au monde. » Cependant, en juin 2008, Dmitri Medvedev décide de mettre fin à cette idée, certes attrayante, mais utopique par définition, en disant que « la seule île de stabilité qui reste dans le monde actuel est l'Antarctique » (колЕсниченко, 2008). Toutefois le Président avance en même temps le projet, plus pragmatique, de faire de la Russie un centre mondial de la finance, sans y trouver de contradiction avec le rejet de l'idée de l'île.

Cette rhétorique offensive complétée par les déclarations de Vladimir Poutine à la fin de 2008 remettant en cause l'entrée de la Russie dans l'Organisation mondiale du commerce, poursuit plusieurs objectifs. D'une part, l'année 2008 marque les dix ans depuis la crise 
russe de 1998, et l'évocation trop fréquente des problèmes liés à la crise actuelle, après dix ans d'une relative stabilité, met à mal la réputation et l'image des hommes politiques en charge de cette stabilité. D'autre part, en détournant l'attention de la société sur la crise hors-frontières, le pouvoir fait adopter des amendements aux lois institutionnelles qui prolongent la durée des mandats présidentiel et parlementaire, s'assurant de cette manière un avenir pérenne. Ce n'est donc qu'après avoir conduit les réformes institutionnelles à leur terme, et après les mesures de sauvetage des banques, que le pouvoir peut se permettre de reconnaitre officiellement l'existence de la crise.

Naturellement, ces manœuvres du pouvoir n'échappent pas aux journalistes de la presse qui tantôt se montrent critiques, tantôt prennent un ton ironique. À partir de l'été 2008 un certain décalage s'établit entre le discours politique et la presse. Dans le premier, on n'évoque la crise qu'en rapport avec les pays occidentaux, principalement, les États-Unis, et l'influence néfaste de leur système financier sur les marchés russes. Dans le second, la crise en Russie est rendue publique, avec un accent mis sur l'inflation, la récession et les aides massives du gouvernement aux banques sous couvert d'un fonds de réserve, accumulé depuis 2001 grâce à la vente des matières premières (БУТРИН, НЕТРЕБА, 2008).

D'une manière générale, l'évolution de la problématique de la crise dans le discours politique est soumise à une analyse critique par la presse qui permet d'avoir une vision globale des manœuvres des autorités.

L'année 2009 se caractérise par une baisse généralisée dans tous les secteurs économiques, mais aussi par la diminution du nombre de milliardaires russes. Cependant, la récession ralentit à partir de mars 2009. Vers la fin de l'année, les résultats des mesures anticrises du gouvernement permettent à Vladimir Poutine d'affirmer que la phase active de la crise est terminée (САВиныХ, 2009). Cela n'épargne pas aux dirigeants politiques certaines manifestations sociales, comme celle de la ville Pikalevo, dans la région de Leningrad, dont les habitants ont bloqué une route d'importance fédérale pour protester contre les licenciements massifs.

Enfin, en 2010 les propos du premier ministre Poutine sur la fin de la crise sont confirmés par le nouveau rapport de la Banque mondiale dont les experts prédisent une hausse du PIB en Russie de $5,9 \%^{8}$. Nous pouvons alors également constater la diminution de l'utilisation dans la presse des «mots de la crise». Ce sont ces apports linguistiques de la crise qu'il nous reste maintenant à examiner.

\section{Les mots de la crise dans le discours de la presse}

Plusieurs principes de classement semblent pertinents pour notre étude des mots de la crise. Cependant, pour revenir aux questionnements du départ, on se limitera à la distinction entre emprunts-néologismes récents et ceux déjà existants dans le langage financier, pour enfin trouver d'éventuels mots-repères relatifs à cette crise précise. Étant donné l'emprise très forte des anglicismes, se pose la question des modes de formation des mots de la crise : calque ou transcription ? Le classement par mode de formation nous permettra d'évaluer mieux la fréquence d'utilisation des mots, mais aussi les "préférences» de la langue pour tel ou tel mode de formation. Certes, le degré d'appropriation des emprunts par la langue ne dépend pas uniquement des modes de formation. Un calqué n'est pas forcément plus productif ou plus couramment employé qu'une transcription. Cependant, ce classement demeure le seul moyen de suivre le passage d'un emprunt dans la langue russe. 
Les emprunts sous forme de calques ou traduction explicite représentent une nette majorité parmi les mots de la crise sélectionnés à partir de l'anglais. Certains peuvent comporter des pièges en raison de la polysémie ou de leur emploi métaphorique développé. C'est le cas de l'expression «bulle financière, immobilière, etc. » (bubble en anglais). L'équivalent russe «Пузырь» s'avère polysémique. Dans les recherches d'emploi de ce terme la polysémie peut nous amener aussi bien vers des textes économiques que médicaux, car le mot russe signifie aussi «la vessie ». Ce mot n'est pas entièrement nouveau dans le russe économique et financier et a été souvent employé dans l'expression « la bulle spéculative » (спекулятивный пузырь). Toutefois, pendant la période étudiée (2007-2009), l'emploi de ce mot au sens économique connaît des développements métaphoriques importants. Dans la plupart des cas, les expressions partent de l'image d'une bulle de savon (мыльный пузырь), les qualificatifs " financière ", « immobilière » ou " économique » étant ajoutés d'une manière presque artificielle. Dans certains cas, l'image d'une bulle de savon s'efface à la suite du développement d'une série de métaphores tirées du domaine de la physique.

[...] РЕЗКИЙ РОСТ ЭТОГО ГОДА ГОВОРИТ О "РАЗОГРЕТОСТИ" РЫНКА. ЭТО РАНО ИЛИ ПОЗДНО ПРИВОДИТ К КИПЕНИЮ И ПОЯВЛЕНИЮ "ПУЗЫРЕЙ". [...] ОСТОРОЖНЫЕ СТРАНЫ [...] СВОИ РЫНКИ ОХЛАЖДАЮТ, А МЫ РАДУЕМСЯ РАЗОГРЕВУ. (ДРУЖИНИНА, 2010)

(« La croissance brusque de cette année témoigne du « réchauffement » du marché. Tôt ou tard cela commence à bouillir et fait apparaître les «bulles » [...] Des pays prudents rafraichissent leurs marchés et nous, on est content de ce réchauffement [...]. »)

Le développement de la métaphore est sans doute lié à d'autres calques, qualifiant notamment le marché comme pouvant être " réchauffé » et " rafraîchi » (heating/cooling the market).

L'emploi du mot «bulle » en russe n'est pas limité aux domaines économique et financier, et peut être valable pour définir une situation associée à la spéculation: « ИНФОРМАцИОННЫЙ МЫльнЫЙ ПУЗЫРЬ, Wikileaks - эТО СвОЕГО РОДА МЫЛЬНЫЙ пузырь " (« bulle d'information, Wikileaks est une sorte de bulle de savon »). Aussi fautil, sans doute, prendre avec prudence la fréquence de son utilisation en tant que « mot de la crise» en russe. Les recherches sur Factiva de l'emploi de ce terme dans des configurations différentes montrent une progression assez nette de son emploi en 2008 et 2009 (voir annexe 1).

D'autres anglicismes employés en russe sous forme de calques connaissent des développements métaphoriques moins importants. Cela s'explique probablement par l'absence du signifié dans les réalités économiques russes. Ainsi les expressions «bad bank» et "toxic assets» (плохой БАнк, токсичныЕ АКтивы) se rencontrent essentiellement dans les articles portant sur les problèmes financiers à l'étranger, même si la question de la création en Russie de «bad bank» fait partie de la polémique des économistes et journalistes russes sur les pages des journaux (ИвАновА, 2009). L'emploi de «toxic assets» en russe peut avoir des développements métaphoriques comme " полигон для ядовитых отходов» (un polygone pour les déchets empoisonnés). Cependant, il s'agit d'avantage de «métaphores d'auteur » inventées par des journalistes ou traducteurs pour rendre leurs textes plus vivants, que d'une véritable appropriation de ces expressions par la langue économique (ШИРОКОВ (ПЕР.), БАЛЦЛИ, МАЛЕР, РОЙТЕР, 2009). Ces néologismes récents sont pourtant assez fréquemment employés dans la presse durant la période étudiée, comme on le voit dans les graphiques (voir annexe 2). 
Le pic de fréquence correspond à l'année 2009 dans les deux cas, car c'est cette année-là que les économistes russes envisageaient la création de ce genre de banque comme une solution aux problèmes du secteur bancaire.

La transcription des anglicismes intervient généralement lorsque le mot emprunté correspond à deux critères : sa forme phonétique est assez simple et il n'y a aucune ambigüité de prononciation ; son contenu est trop complexe pour être calqué ou traduit de manière compacte. Ainsi, des anglicismes comme Recession, Hedge funds, Derrivative, Volatility, Swap, Subprime sont employés dans le russe économique et financier en transcription et ont le même sens qu'en anglais (РЕЦЕССИЯ, ХЕДЖ-ФОНДЫ, ДЕРРИВАТИВЫ, ВОЛАТИЛЬНОСТЬ, СВОПЫ, САБПРАЙМ/СУБПРАЙМ/subprime).

L'appartenance stricte de ces emprunts au langage financier n'exclut pourtant pas, dans certains cas, une appropriation plus profonde d'une transcription que d'un terme calquée . Parmi nos exemples, le candidat le plus prometteur au déracinement linguistique est l'expression hedge fund qui se montre suffisamment productive pour donner lieu à des adaptations et jeux de mots en russe. Ainsi, on l'emploie en faisant allusion à une chanson populaire des années 1960 : « ЖИЛ ДА БЫЛ чЕРНЫЙ ХЕДЖ ЗА БУГРОМ » - « Il était une fois un hedge noir à l'étranger » (ЗУБАКОВ, 2008). Il peut arriver qu'on s'amuse à fusionner ce mot avec d'autres, afin de combiner leurs connotations, comme dans «эмИРАТЫ ЗАХАДЖИРОВАЛИ РИСКИ» (ХВОСТИК, ГАЙДАЕВ, 2009). Ici, dans un article présentant la situation sur le marché financier des Émirats Arabes, la combinaison des mots « ХЕДЖ » et «ХАДж» (hedge / hajj), phonétiquement très proches en russe, joue un rôle d'animation informative.

En revanche, le terme subprime semble être plus difficile à assimiler par le russe. Ainsi son emploi sous forme de transcription s'avère beaucoup plus rare que l'emploi tel quel, en anglais, suivi d'une explication en russe. Les deux façons de transcrire ce mot en russe ( СУБПРАЙМ et САБПРАЙМ) ne représentent que 29 et 35 occurrences entre 2007 et 2009, à comparer avec les 1323 cas d'emploi du même mot en anglais (voir annexe 3). La baisse significative de l'emploi de ce terme s'explique par un très faible enracinement dans la langue et la vie économique russes de la notion américaine.

Les données de Factiva nous montrent, à quelques détails près, une hausse sensible de l'emploi de ces mots dans la presse russe entre 2007 et 2009 et un net ralentissement vers 2010. Cela nous permet de les associer à la catégorie des «mots de la crise ", comme en anglais. Ceci étant, la fréquence de leur emploi est étroitement liée aux événements sur les marchés financiers mondiaux et ne reflète pas entièrement la situation spécifique de la Russie. Nous avons par ailleurs constaté la flambée pendant la même période de l'emploi des autres termes du langage financier, par exemple «свопо пционы, ДЕРРИВАТИВЫ, ФьючЕРСЫ ». Cela s'explique par l'augmentation des flux d'informations relatives à la situation des marchés en Russie et dans les autres pays.

En revanche, la crise a contribué aux changements sémantiques de la terminologie de la finance. C'est notamment le cas de hedge funds dont le sens d'origine, aux connotations positives et sécurisantes, s'est transformé, aussi bien en anglais qu'en russe, jusqu'à désigner pratiquement son contraire. Pour le russe, cette période est aussi caractérisée par l'arrivée d'un certain nombre de néologismes et par le développement du sens des expressions existantes. Cela nous amène à formuler quelques conclusions sur les représentations de la crise et son impact sur la langue. 


\section{Conclusions}

L'étude des mutations de la langue russe pendant la crise économique de 2007-2009 nous permet de considérer la presse généraliste-experte comme à la fois une source assez flexible par lequel le russe économique et financier s'enrichit des néologismes relatifs à la crise et un moyen de transfert de ces néologismes dans le langage courant. Toutefois, à la différence des crises de 1992 et 1998, en 2007-2009 la presse demeure la seule source et le seul terrain d'essai des emprunts-anglicismes, car les dirigeants politiques évitent de les employer fréquemment, montrant de cette manière que cette nouvelle crise n'a pas ses origines en Russie, mais bel et bien en Occident. C'est donc leur façon de se distancier des pays occidentaux. Cela explique probablement l'absence en russe des mots-repères relatifs à cette crise, contrairement à 1992 et 1998. Les néologismes de cette crise, parce qu'ils ne sont pas liés à la personne d'un dirigeant, n'entrent pas d'une manière durable dans le langage courant afin de conserver certaines notions de la crise dans la mémoire linguistique collective, et leur emploi se limite aux domaines économique et financier. Certes, il est encore prématuré de conclure sur le degré d'appropriation des néologismes récents par la langue. Cependant, la fréquence d'emploi des emprunts-néologismes dans la presse ne peut avoir que peu d'incidence sur l'élargissement de son usage dans les domaines autres que la finance.

Dans ses représentations de la crise, la presse généraliste-experte tente de polémiquer avec le pouvoir sans pourtant se permettre une critique ouverte des mesures gouvernementales. Son rôle se résume davantage à des tentatives d'alerter le pouvoir et l'opinion publique sur les dangers potentiels, mais elle se garde de semer la panique parmi la population. Cela correspond d'ailleurs aux objectifs des gouvernants dont le discours s'efforce de minimiser les conséquences de la crise. C'est probablement ce caractère ambigu du discours de la presse russe qui expliquerait un impact assez faible des néologismes journalistiques sur leur emploi dans le langage courant.

Enfin, la similitude des pics de fréquence d'emploi des mots de la crise en russe, en anglais et dans les autres langues confirmée lors de journée d'études de l'ILCEA sur « Traduire la crise : 2007-2009", témoigne d'une certaine uniformisation du lexique et des métaphores dans le domaine de l'information économique liée, sans doute, à la globalisation accrue de l'économie mondiale.

\section{BIBLIOGRAPHIE}

Russian Economic Report, $\mathrm{n}^{\circ}$ 16, juillet 2008, disponible sur le site de la Banque Mondiale <http:// www-wds.worldbank.org>

Russian Economic Report, $\mathrm{n}^{\circ}$ 21, mars 2010, disponible sur le site de la Banque Mondiale <http:// www-wds.worldbank.org>

АСКЕР-ЗАДЕ Н., «ДО НАС ДОШЛО», КОММЕРСАНТ, 17 janvier 2008. 
БОРОДИН А., « ЗАЧЕМ СОЗДАВАТЬ ПЛОХИЕ БАНКИ? ДАВАЙТЕ СОЗДАВАТЬ И РАЗВИВАТЬ ХОРОШИЕ», МАТЕРИАЛЫ ПРЕСС-КОНФЕРЕНЦИИ АНАЛИТИЧЕСКИЙ БАНКОВСКИЙ ЖУРНАЛ, 30 mars 2009. БУТРИН Д. et НЕТРЕБА П., «КРИЗИС ЗАВАЛИВАЮТ ДЕНЬГАМИ», КОММЕРСАНТ, 17 octobre 2008 БУТУРЛИН Д. et ШАПОВАЛОВ А., «ДМИТРИЙ МЕДВЕДЕВ ДОКОПАЛСЯ ДО КОРНЕЙ ФИНАНСОВОГО КРИЗИСА», КОММЕРСАНТ, 9 juin 2008.

ДРУЖИНИНА А., «АКЦИИ БЕЗ ЧУДЕС», НОВЫЕ ИЗВЕСТИЯ, 23 décembre 2010.

ЗУБАКОВ В., «ЖИЛ ДА БЫЛ "ЧЕРНЫЙ ХЕДЖ" ЗА БУГРОМ...», ВРЕМЯ И ДЕНЬГИ, 2 juillet 2008.

ИВАНОВА Е., «ПЛОХОЙ БАНК. СДЕЛАНО В РОССИИ», ДЕЛОВОЙ ПЕТЕРБУРГ, 7 mai 2009.

ИлЛАРИОНОВ А., «ЧТО ТАКОЕ- КРИЗИС?», TheNewTimes, nº40(86), 6 octobre 2008, с.4

КАЛЕДИНА А. et ЧАЙКА Ф., «КАКОГО КРИЗИСА НАМ БОЯТЬСЯ?», ИЗВЕСТИЯ, 13 février 2008.

КОЛЕСНИЧЕНКО А., «МЕДВЕДЕВ: В МИРЕ ОСТАЛСЯ ОДИН ОСТРОВ СТАБИЛЬНОСТИ - АНТАРКТИДА», АИФ, 5 juin 2008.

КОЧЕВА О. еt ЧУВИЛЯЕВ П., «ВОЙНА ПРОТИВ ЧЕРНОГО ВТОРНИКА», ДЕНЬГИ, $\mathrm{n}^{\circ}$ 8, 2007.

МИНАЕВ С., «ТОВАРНО-ФРОНТОВЫЕ ОТНОШЕНИЯ», ВЛАСТЬ, 18 aоût 2008.

САВИНЫХ А., «ПРАВИТЕЛЬСТВУ ПРИКАЗАНО НЕ РАССЛАБЛЯТЬСЯ», ИЗВЕСТИЯ, 30 décembre 2009.

ЧАЙКА Ф., «РОССИЯ ПРОТЯГИВАЕТ МИРУ РУКУ ПОМОЩИ», ИЗВЕСТИЯ, 24 janvier 2008.

ЧувиЛЯЕВ П., «МЕДВЕЖЬИ ГРЁЗЫ», ДЕНЬГИ, nº 9, 2007.

ШИРОКОВ В. (ПЕР.), БАЛЦЛИ Б., МАЛЕР А. еt РОЙТЕР В., «МНОГО ДЕНЕГ, МАЛО КАПИТАЛА», ПРОФИЛЬ, 6 juillet 2009.

ХВОСТИК Е. еЕ ГАЙДАЕВ В., «ЭМИРАТЫ ЗАХАДЖИРОВАЛИ РИСКИ», КОММЕРСАНТ, 27 novembre 2009.

\section{ANNEXES}

\section{Annexe 1.}


Bulle - Пузырь.

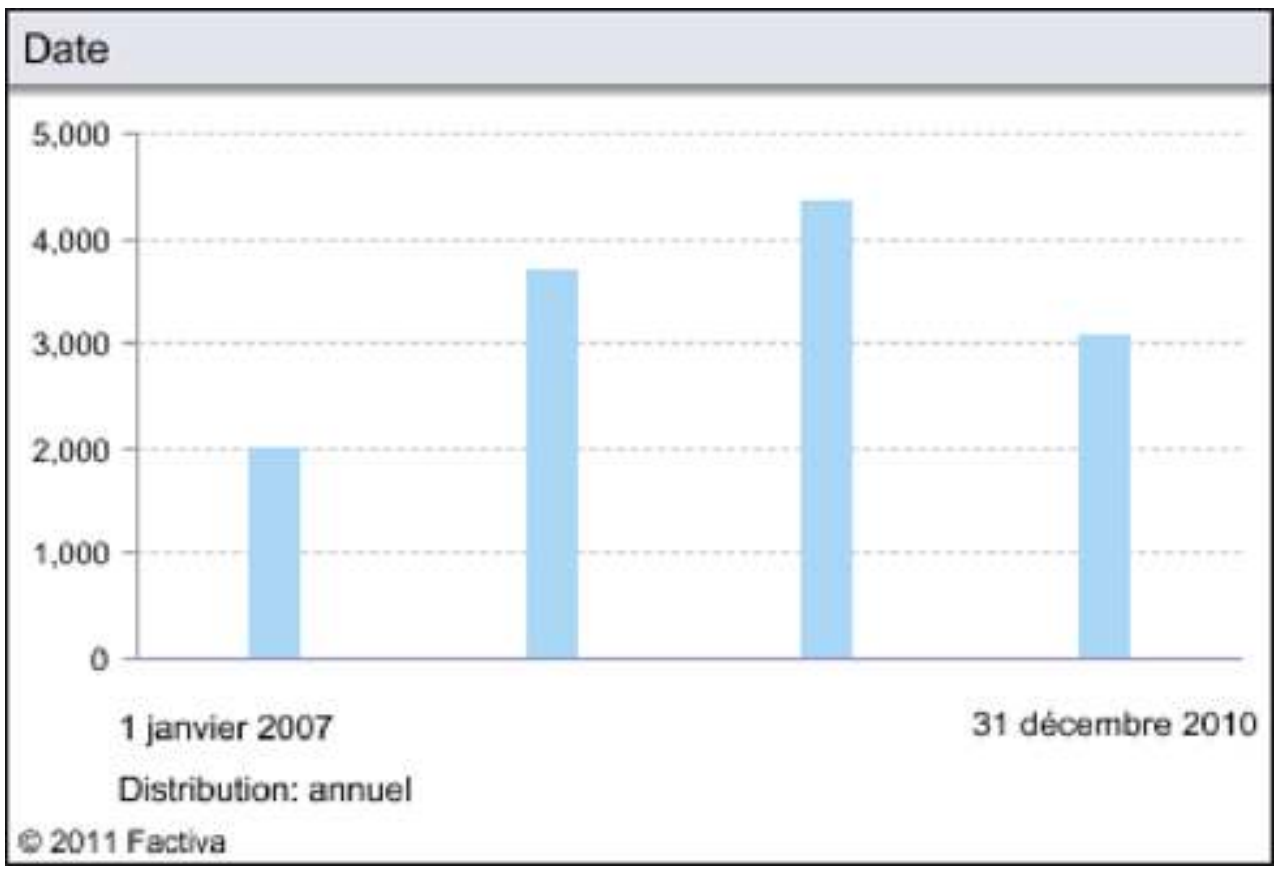

Bulle de savon - Мыльный пузырь.

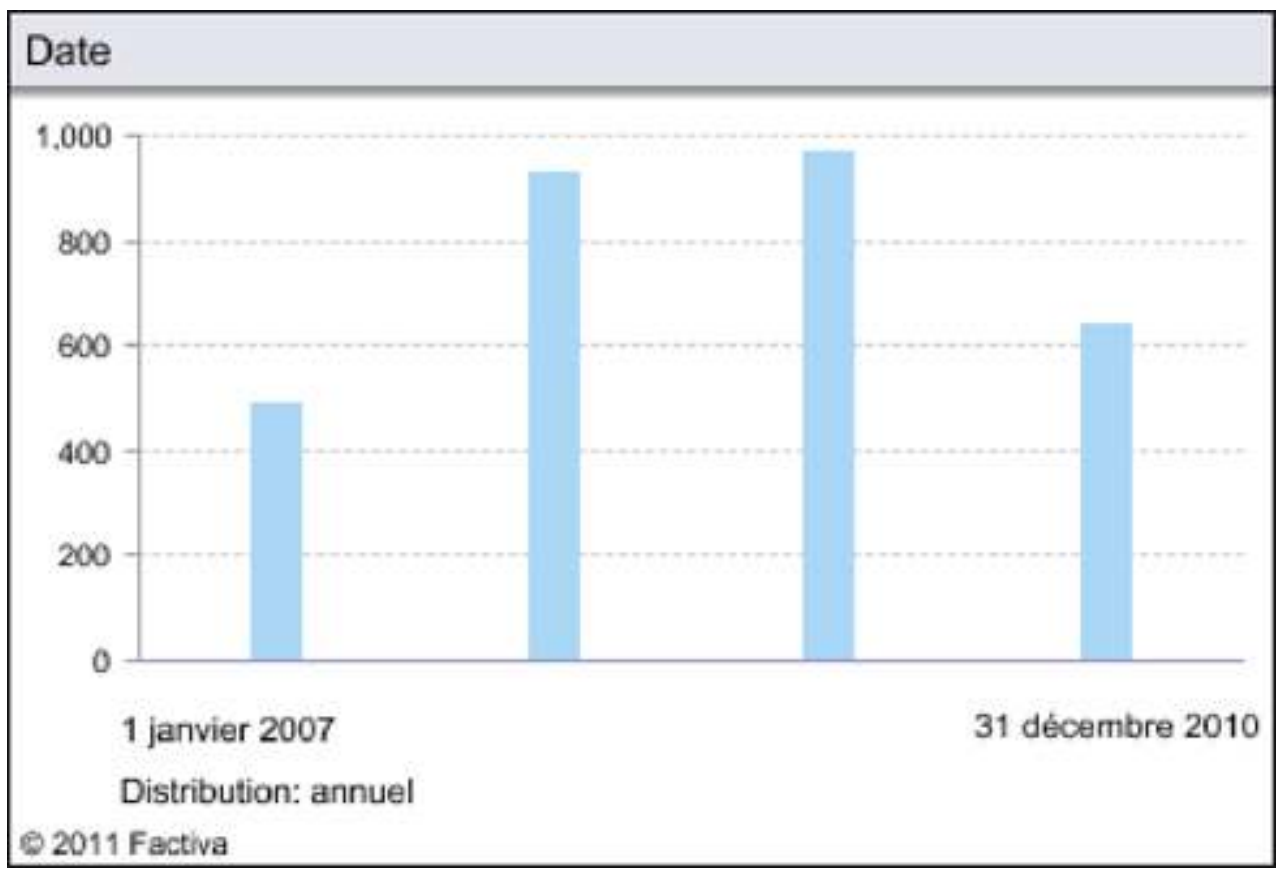


Bulle économique - Экономический пузырь.

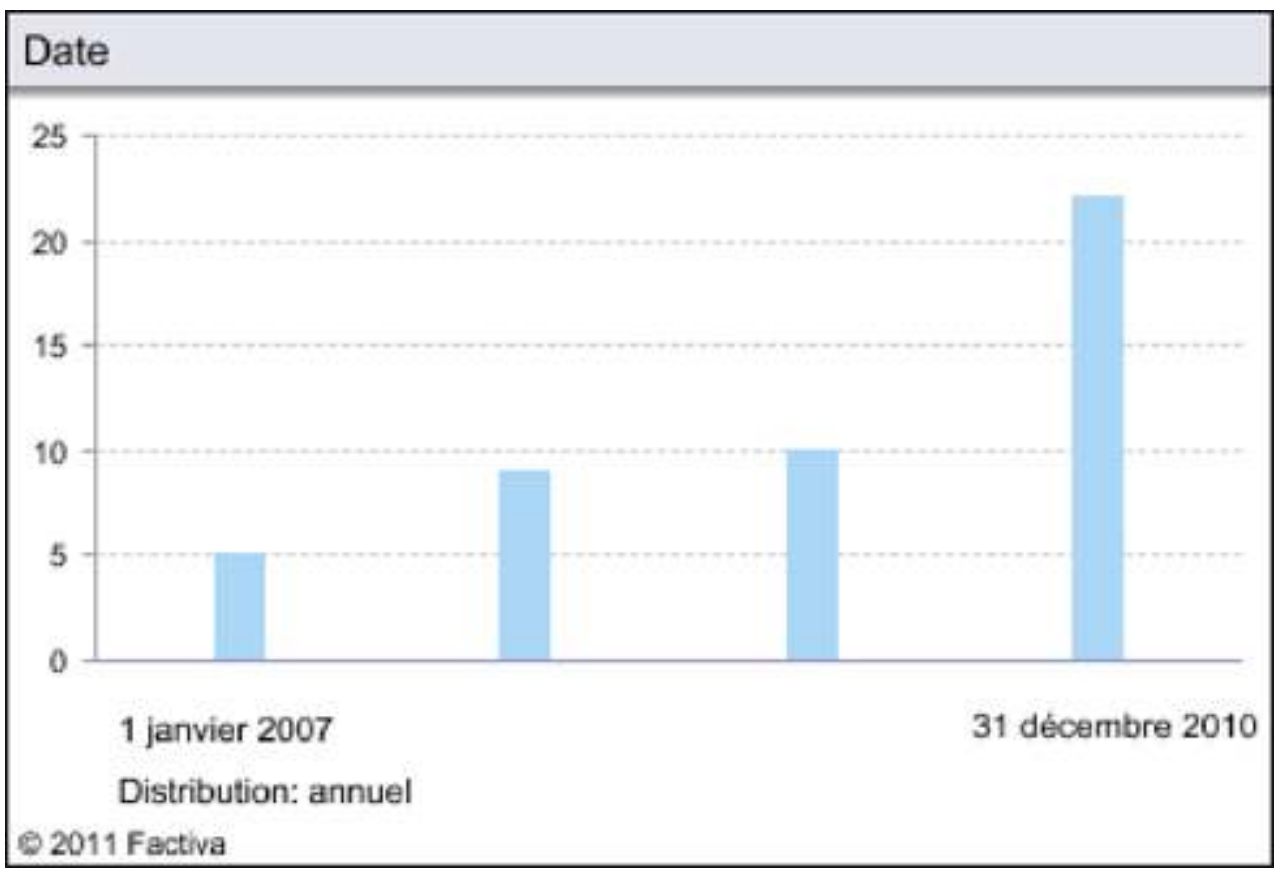

\section{Annexe 2.}

Bad bank - Плохой банк.

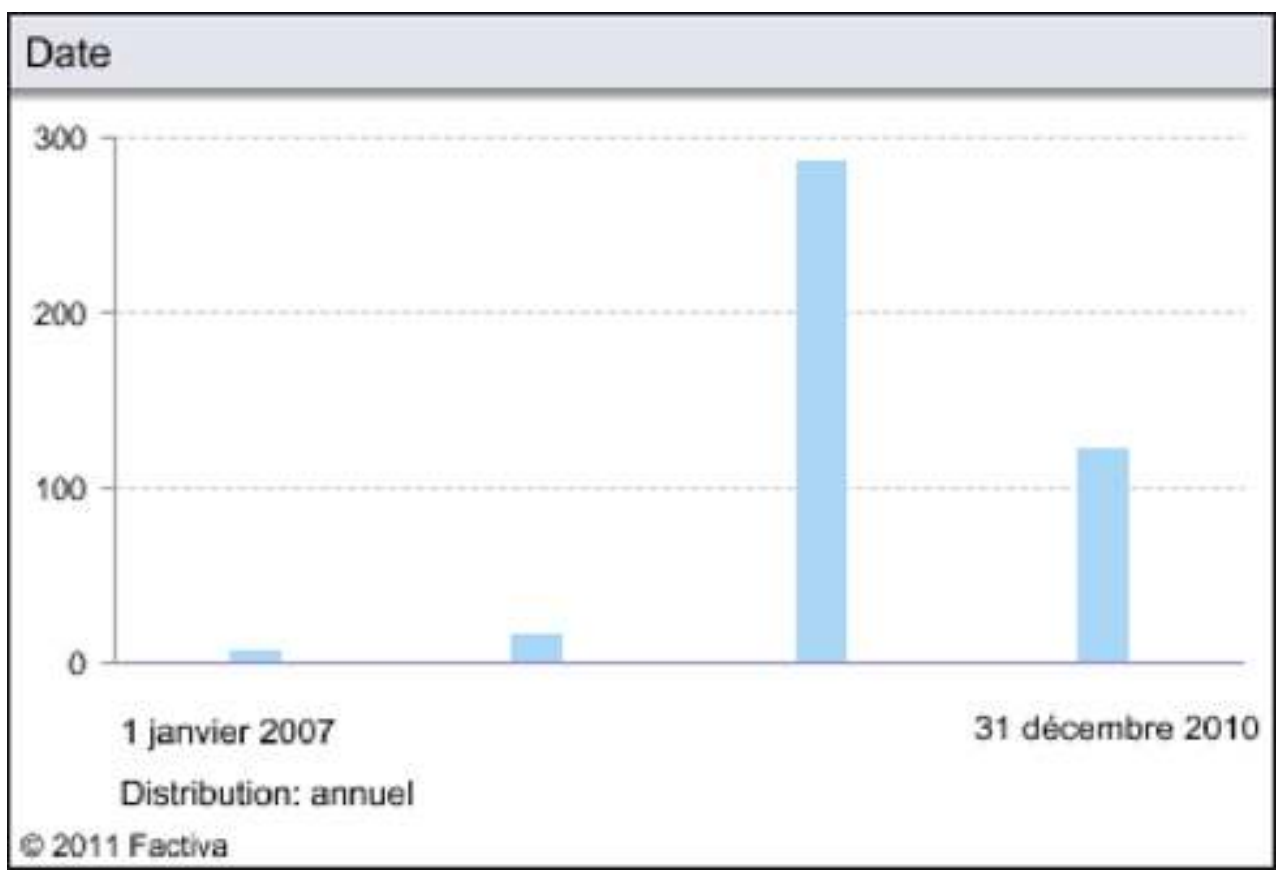


Toxic assets - Токсичные активы.

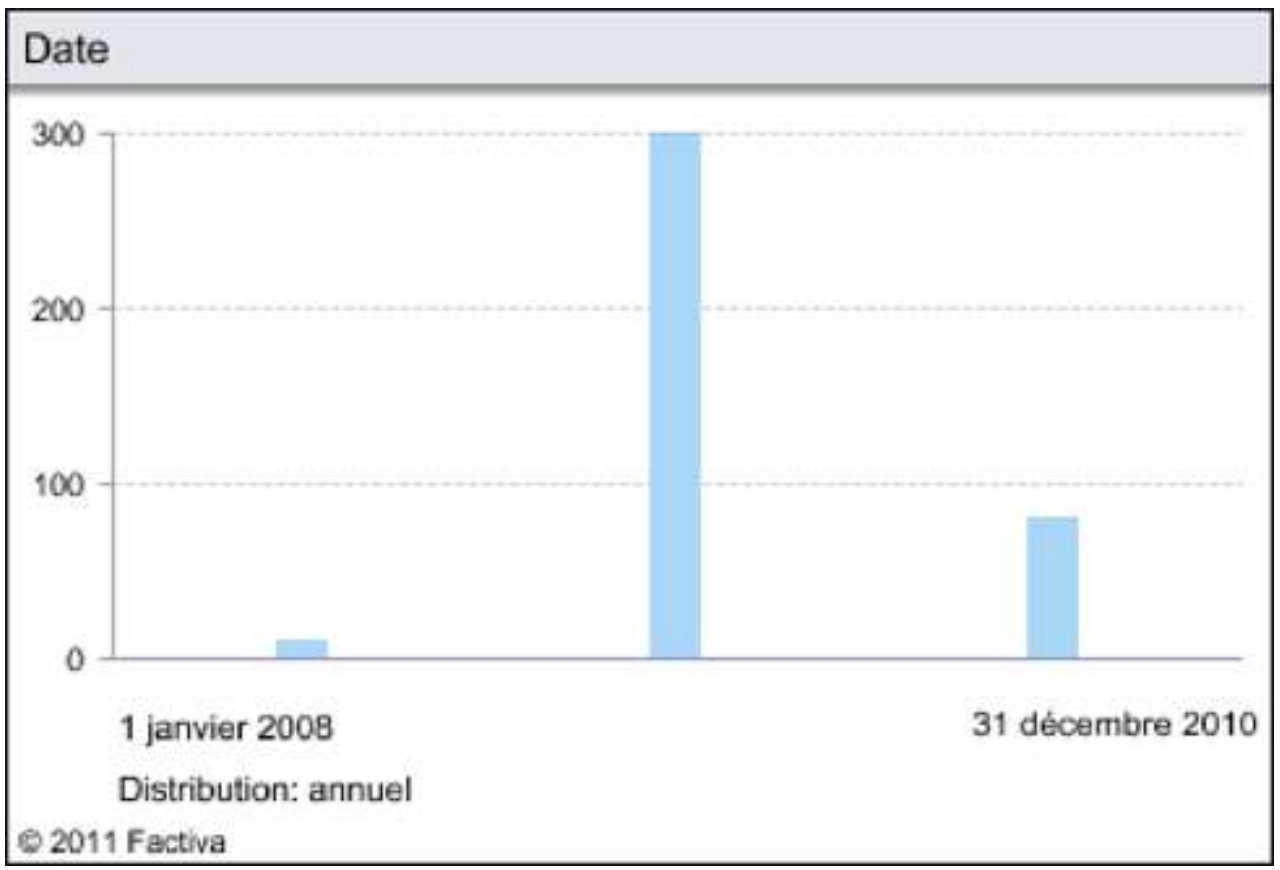

\section{Annexe 3.}

Hedge fund - Хедж-фонд.

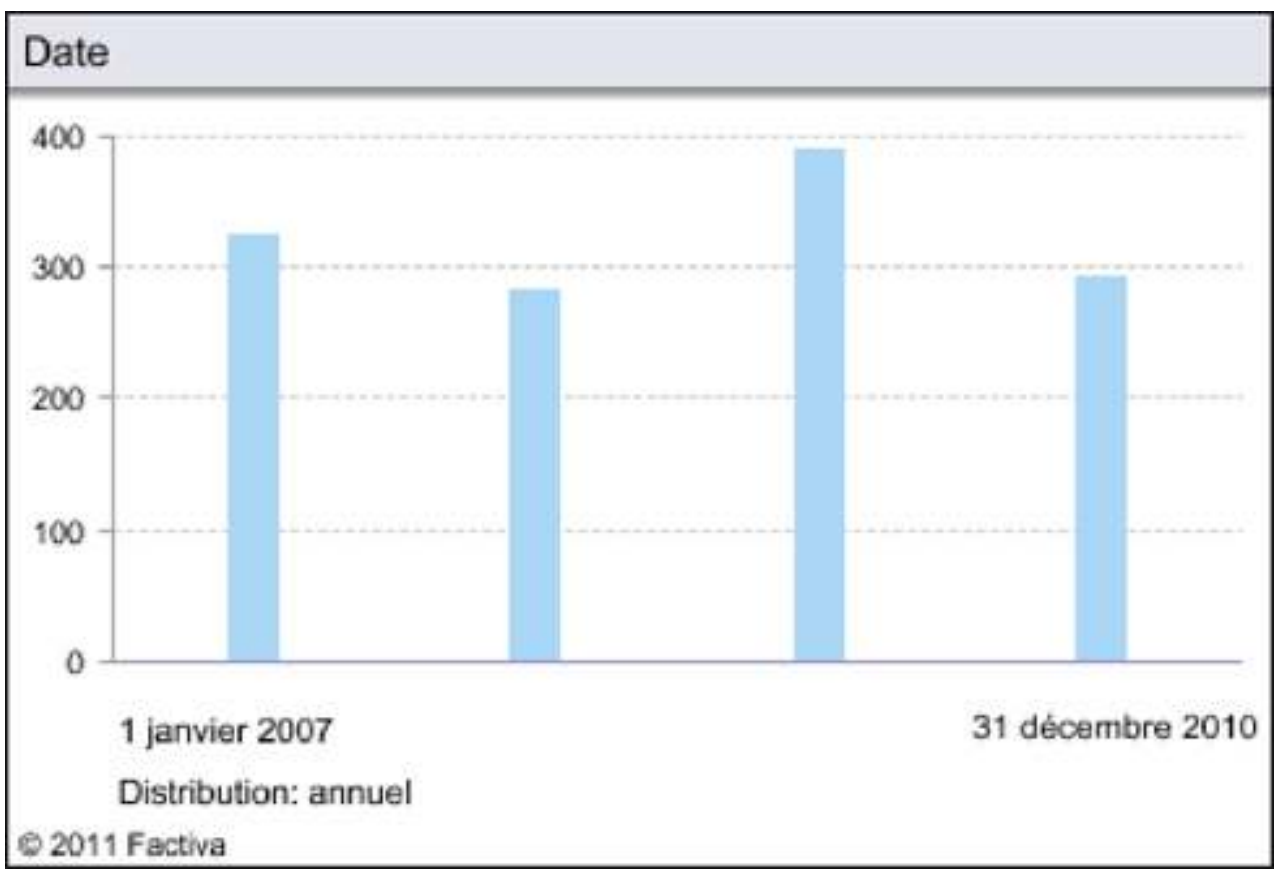




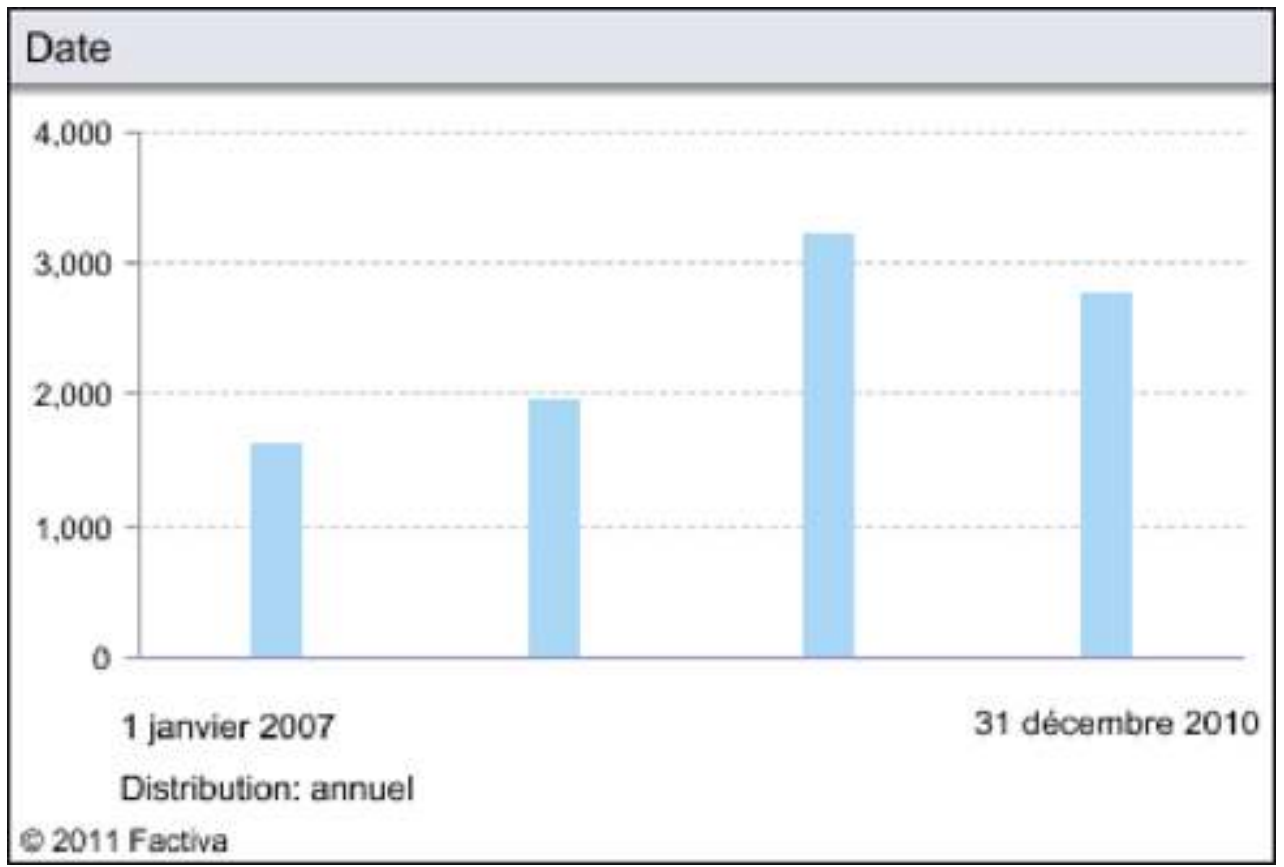

Subprime (version anglaise).

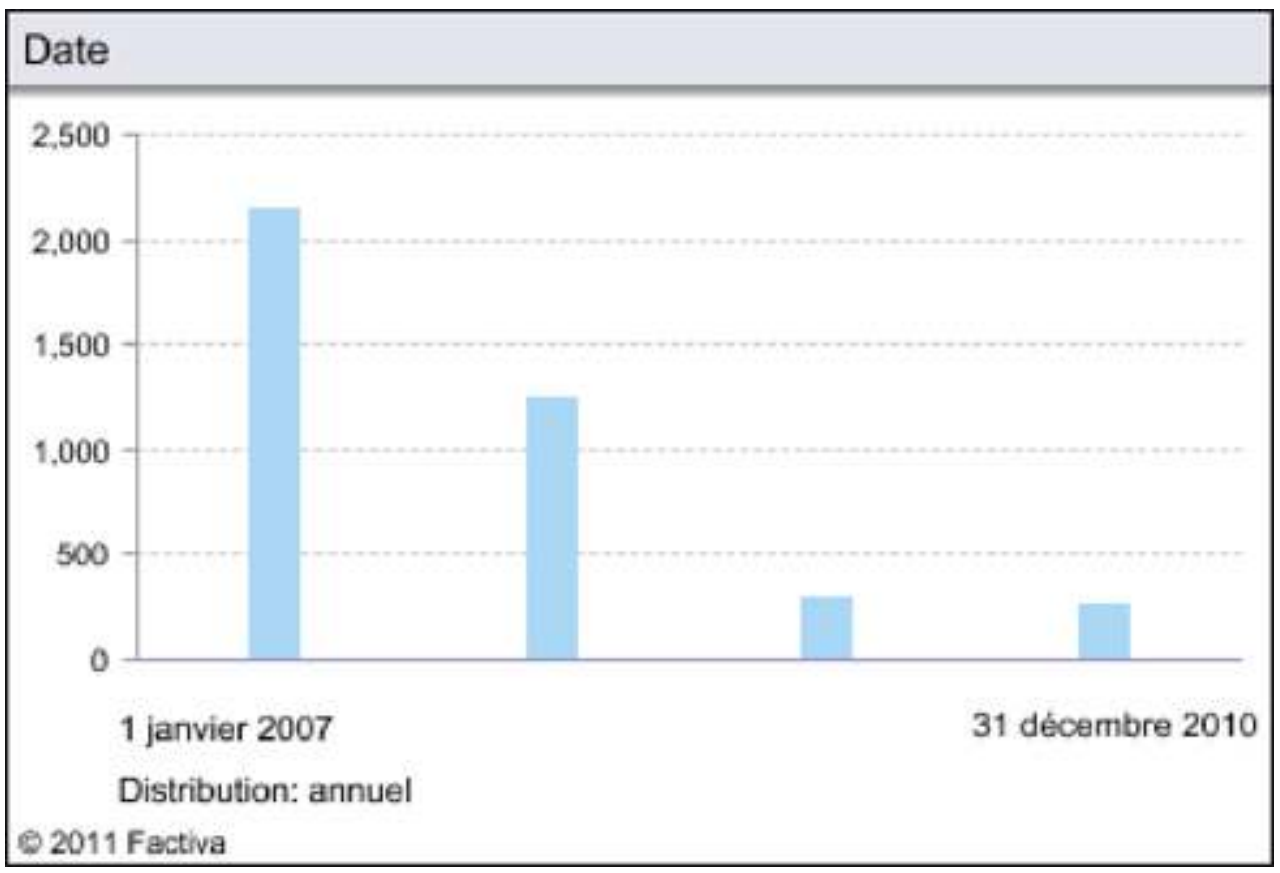

\section{NOTES}

1. Journée d'études de l'ILCEA, « La crise », juin 2010, université Stendhal, Grenoble (France).

2. Ce corpus provient essentiellement de la base de données en ligne Factiva. Il est complété par un choix d'articles de la presse du même type. L'ensemble des articles couvre la période du 1 janvier 2007 au 31 décembre 2010. 
3. L'expression «les mots de la crise ", c'est-à-dire ceux qui sont fréquemment utilisés pour décrire la crise financière dans les langues faisant partie de notre projet (l'anglais, l'allemand, l'espagnol, l'italien et le russe) ainsi que le choix de ces mots ont été établis en commun avec l'équipe multilingue de l'ILCEA/GREMUTS de l'université Stendhal, Grenoble.

4. La traduction en français des expressions et des citations russes a été faite par l'auteur de l'article.

5. Le titre de cet article en russe comporte un jeu de mots : до нАС дошло signifie à la fois "c'est parvenu jusqu'à nous" et "nous avons pigé".

6. En réalité, ce qu'on présente comme nouveau est souvent de l'ancien bien oublié et légèrement corrigé en fonction de l'époque et des circonstances. En effet, les exemples de déclarations de ce type sont assez fréquents dans l'histoire soviétique, à commencer par le Décret sur la paix des bolchéviques en 1917, en passant par les thèses de Staline sur la victoire du socialisme suivie de la baisse, très sélective, des prix alimentaires dans les années 1930, pour finir avec le fameux projet de Khrouchtchev à la fin des années 1950 de «construire le communisme » pour les années 1980.

7. Russian Economic Report, $\mathrm{n}^{\circ}$ 16, juillet 2008, disponible sur le site de la Banque Mondiale $<$ http://www-wds.worldbank.org>

8. Russian Economic Report, $\mathrm{n}^{\circ} 21$, mars 2010, disponible sur le site de la Banque Mondiale <http:// www-wds.worldbank.org>

9. L'exemple le plus courant en est le sigle PR (public relations) qui a été rapidement russifié dans les dix dernières années, au point même que son origine anglaise semble presque oubliée.

\section{RÉSUMÉS}

Faisant partie du projet collectif du GREMUTS-ILCEA (voir éditorial) le présent article poursuit, d'une part, l'objectif général consistant à étudier le rapport entre la crise économique récente (2007-2009) et le lexique économique ou «les mots de la crise » dont l'emploi dans la presse internationale s'intensifie au fur et à mesure que la crise s'aggrave. D'autre part, il est également question de l'impact de la crise en Russie sur l'apparition dans la langue russe d'un certain nombre de néologismes propres à la thématique de la crise. La problématique des « mots de la crise » sera donc abordée sous deux angles, à savoir du point de vue de la fréquence de leur emploi dans une perspective comparative avec d'autres langues et en suivant une démarche fondée sur l'analyse des discours politique et médiatique russes qui permettrait de définir leur influence sur la langue et son enrichissement par des néologismes relevant du domaine économique.

As a part of the collective GREMUTS/ILCEA project (see editorial), this article aims at studying, on the one hand, the connection between the recent economic crisis (2007-2009) and the economic lexicon or "words of the crisis" that are increasingly used in the international press with the intensification of the crisis. On the other hand, the article also analyses the question of how the crisis in Russia impacts the creation of a variety of neologisms clearly situated in the field of this particular theme. The question will be studied from two perspectives, firstly, the frequency at which they are used seen in a comparative perspective against other languages and, secondly, discourse analysis approach to Russian political and media discourse with a view to 
enabling us to assess their influence on language and its enrichment through neologisms related to the field of economics

INDEX

Mots-clés : crise économique, discours politique, néologismes, Russie

Keywords : economic crisis, neologism, political discourse, Russia

\section{AUTEUR}

VALÉRY KOSSOV

MCF, Université Stendhal - Grenoble 3. ILCEA 